\title{
27. Free vibration analysis of electric-magneto-elastic functionally graded plate with uncertainty
}

\author{
G. Q. Xie ${ }^{1}$, J. H. Tian ${ }^{2}$ \\ ${ }^{1}$ Civil Engineering College, Hunan University of Science and Technology, Xiangtan, 411201, China \\ ${ }^{2}$ School of Mechatronic Engineering, Xi'an Technological University, Xi'an, 710032, China \\ ${ }^{1}$ Corresponding author \\ E-mail: ${ }^{1} 1020095 @$ hnust.edu.cn, ${ }^{2540673737 @ q q . c o m}$
}

Received 27 October 2016; accepted 28 October 2016

DOI https://doi.org/10.21595/mme.2016.17891

\begin{abstract}
Free vibration analysis of electric-magneto-elastic functionally graded plate with uncertain parameters is studied in this paper. The elastic, electric and magnetic parameters are regarded as interval variables. Interval analysis of electric-magneto-elastic functionally graded plate are carried out by introducing the interval algorithm into the dynamic equations. A simply supported electric-magneto-elastic functionally graded plate as a numerical example are provided to the validity of the method.
\end{abstract}

Keywords: free vibration, electric-magneto-elastic functionally graded plate, uncertainty, interval algorithm.

\section{Introduction}

Electric-magneto material is a new intelligent material transforming energy from one to the other (among magnetic, electric and mechanical energy). Applied in ultrasonic imaging devices, sensors, actuators, transducers and many other emerging components, they have attracted wide and increasing attention to their static and dynamic behaviors in recent decades [1-7]. However, parameters of the studied electric-magneto-elastic structures used to consider as deterministic ones [8-10]. Indeed, it is very difficult to determine accurately the magnetic and electric parameters because errors will arise when they are manufactured or measured, specially the magneto-electric coefficient of mutual induction.

There are a lot of researches on interval static and dynamic analysis of structure, Han and Jiang et al. [11] dealt with the wave propagation problems in composite-laminated plates subjected to uncertainty in load and material property combined the interval analysis method with the hybrid numerical method (HNM). Li, Luo and Sun [12] studied the reliability-based multiobjective optimization by using a new interval strategy to model uncertain parameters. Jiang and Han et.al [13] proposed a method to solve the nonlinear interval number programming problem with uncertain coefficients both in nonlinear objective function and nonlinear constraints. Based on an order relation of interval number, the uncertain objective function is transformed into two deterministic objective functions, in which the robustness of design is considered. Kang, Luo and Li [14] investigated the formulation and numerical solution of reliability-based optimization of structures exhibiting grouped uncertain-but-bounded variations. $\mathrm{Wu}$, Zhao and Chen [15] proposed an improved interval analysis method for uncertain structures.

\section{Dynamic equations}

The coupling physic equations for anisotropic and linear electric-magneto-elastic solids are given by:

$\left\{\begin{array}{l}\sigma_{i j}=C_{i j k l} \varepsilon_{k l}-e_{i j m} E_{m}-q_{i j n} H_{n}, \\ D_{m}=e_{i j m} \varepsilon_{i j}+g_{m n} E_{n}+\alpha_{m n} H_{n}, \\ B_{n}=q_{i j n} \varepsilon_{i j}+\alpha_{m n} E_{m}+\mu_{m n} H_{m}, \quad i, j, m, n=1,2,3,\end{array}\right.$ 
where $\sigma_{i j}$ denotes stress tensor, $\varepsilon_{k l}$ strain tensor, $D_{m}$ the electric displacement tensor and $B_{m}$ the magnetic induction tensor, $C_{i j k l}, g_{m n}$ and $\mu_{m n}$ are the elastic, dielectric and magnetic permeability coefficient tensors. $q_{i j n}, e_{i j m}$ and $\alpha_{m n}$ are piezoelectric, piezomagnetic and magneto-electric material coefficient tensors.

Tenor forms of the generalized geometric equations are given by:

$$
\begin{cases}\varepsilon_{i j}=\frac{1}{2}\left(u_{i, j}+u_{j, i}\right), & i, j=1,2,3, \\ E_{i}=-\varphi_{, i}, & i=1,2,3, \\ H_{i}=-\psi_{, i}, & i=1,2,3,\end{cases}
$$

where $u$ is the elastic displacement, $\varphi$ is the electric potential, $\psi$ is the magnetic potential. The comma in the subscript denotes partial derivative.

The governing equations of electric-magneto-elastic functionally graded plate, absent of the body force, electric charge density, and electric current density, are given by:

$$
\left\{\begin{array}{l}
\sigma_{i j, j}=\rho \ddot{u}_{i}, \\
D_{i, i}=0, \\
B_{i, i}=0 .
\end{array}\right.
$$

The functionally graded material parameters are assumed to obey exponential law across the thickness direction ( $z$ axis):

$$
\begin{aligned}
& C_{i j k l}=C_{i j k l}^{0} e^{\eta z}, \quad g_{i j}=g_{i j}^{0} e^{\eta z}, \quad \mu_{i j}=\mu_{i j}^{0} e^{\eta z}, \\
& q_{i j l}=q_{i j l}^{0} e^{\eta z}, \quad e_{i j l}=e_{i j l}^{0} e^{\eta z}, \quad \alpha_{i j}=\alpha_{i j}^{0} e^{\eta z}, \quad \rho=\rho^{0} e^{\eta z},
\end{aligned}
$$

where $\eta$ is the exponential factor characterizing the degree of the material gradient in the $z$-direction, and the superscript 0 is attached to indicate the material coefficients for $z=0$. It is obvious that $\eta=0$ corresponds to the homogeneous material case.

For a special case, an orthotropic transverse isotropic solid, the material coefficients in Eq. (1) can be written as:

$$
\begin{aligned}
& {[C]=\left[\begin{array}{llllll}
C_{1111} & C_{1122} & C_{1133} & 0 & 0 & 0 \\
& C_{2222} & C_{2233} & 0 & 0 & 0 \\
s y m & & C_{3333} & 0 & 0 & 0 \\
& & & C_{2323} & 0 & 0 \\
& & & C_{3131} & 0 \\
0 & & & C_{1212}
\end{array}\right], \quad[e]=\left[\begin{array}{ccc}
0 & 0 & e_{113} \\
0 & 0 & e_{223} \\
0 & 0 & e_{333} \\
0 & e_{232} & 0 \\
e_{311} & 0 & 0 \\
0 & 0 & 0
\end{array}\right],} \\
& {[q]=\left[\begin{array}{ccc}
0 & 0 & q_{113} \\
0 & 0 & q_{223} \\
0 & 0 & q_{333} \\
0 & q_{232} & 0 \\
q_{311} & 0 & 0 \\
0 & 0 & 0
\end{array}\right], \quad[g]=\left[\begin{array}{ccc}
g_{11} & 0 & 0 \\
0 & g_{22} & 0 \\
0 & 0 & g_{33}
\end{array}\right], \quad[\alpha]=\left[\begin{array}{ccc}
\alpha_{11} & 0 & 0 \\
0 & \alpha_{22} & 0 \\
0 & 0 & \alpha_{33}
\end{array}\right],} \\
& {[\mu]=\left[\begin{array}{ccc}
\mu_{11} & 0 & 0 \\
0 & \mu_{22} & 0 \\
0 & 0 & \mu_{33}
\end{array}\right] .}
\end{aligned}
$$

For a simply supported and FGM rectangular plates $(a \times b)$, we adopt the solution of the generalized displacement in the form: 


$$
\left\{\begin{array}{l}
u_{1}(x, y, z, t) \\
u_{2}(x, y, z, t) \\
u_{3}(x, y, z, t) \\
\varphi(x, y, z, t) \\
\psi(x, y, z, t)
\end{array}\right\}=e^{-\frac{\eta z}{2}} e^{i \omega t}\left\{\begin{array}{l}
a_{1} \cos (p x) \sin (q y) \\
a_{2} \sin (p x) \cos (q y) \\
a_{3} \sin (p x) \sin (q y) \\
a_{4} \sin (p x) \sin (q y) \\
a_{5} \sin (p x) \sin (q y)
\end{array}\right\},
$$

where $p=m \pi / a, q=n \pi / b$, with $n$ and $m$ are two positive integers.

Substitution of Eq. (6) into Eq. (2), the constitutive Eq. (1), and finally into the governing Eq. (3), yields the following eigenequation:

$$
\left[-\mathbf{A}+\frac{\eta}{2}\left(\mathbf{R}+\mathbf{R}^{T}+\frac{\eta}{2} \mathbf{G}\right)-\omega^{2} \mathbf{M}\right] \mathbf{U}=0
$$

where:

$$
\begin{aligned}
& \mathbf{U}=\left[\begin{array}{lllll}
a_{1} & a_{2} & a_{3} & a_{4} & a_{5}
\end{array}\right]^{T}, \\
& \mathbf{R}=\left[\begin{array}{ccccc}
0 & 0 & p C_{1133}^{0} & p e_{313}^{0} & p q_{313}^{0} \\
0 & 0 & q C_{2233} & q e_{323}^{0} & q q_{323}^{0} \\
-p C_{3131}^{0} & -q C_{2323}^{0} & 0 & 0 & 0 \\
-p e_{311}^{0} & -q e_{231}^{0} & 0 & 0 & 0 \\
-p q_{311}^{0} & -q q_{231}^{0} & 0 & 0 & 0
\end{array}\right], \quad \mathbf{M}=\left[\begin{array}{ccccc}
\rho^{0} & 0 & 0 & 0 & 0 \\
0 & \rho^{0} & 0 & 0 & 0 \\
0 & 0 & \rho^{0} & 0 & 0 \\
0 & 0 & 0 & 0 & 0 \\
0 & 0 & 0 & 0 & 0
\end{array}\right], \\
& \mathbf{G}=\left[\begin{array}{ccccc}
C_{3131}^{0} & 0 & 0 & 0 & 0 \\
s y m & C_{2323}^{0} & 0 & 0 & 0 \\
s y & C_{3333}^{0} & e_{333}^{0} & q_{333}^{0} \\
\mathbf{A} & = & -g_{33}^{0} & -\alpha_{33}^{0} \\
\mathbf{0}_{3 \times 2} & \mathbf{A}_{22}
\end{array}\right], \\
& \mathbf{A}_{11}=\left[\begin{array}{ccc}
-\left(C_{1111}^{0} p^{2}+C_{1212}^{0} q^{2}\right) & -p q\left(C_{1122}^{0}+C_{1212}^{0}\right) \\
-p q\left(C_{1122}^{0}+C_{1212}^{0}\right) & -\left(C_{1212}^{0} p^{2}+C_{2222}^{0} q^{2}\right)
\end{array}\right], \\
& \mathbf{A}_{22}=\left[\begin{array}{cccc}
-\left(C_{3131}^{0} p^{2}+C_{2323}^{0} q^{2}\right) & -\left(e_{311}^{0} p^{2}+e_{232}^{0} q^{2}\right) & -\left(q_{311}^{0} p^{2}+q_{232}^{0} q^{2}\right) \\
-\left(e_{311}^{0} p^{2}+e_{232}^{0} q^{2}\right) & g_{11}^{0} p^{2}+g_{22}^{0} q^{2} & \alpha_{11}^{0} p^{2}+\alpha_{22}^{0} q^{2} \\
-\left(q_{311}^{0} p^{2}+q_{232}^{0} q^{2}\right) & \alpha_{11}^{0} p^{2}+\alpha_{22}^{0} q^{2} & \mu_{11}^{0} p^{2}+\mu_{22}^{0} q^{2}
\end{array}\right] .
\end{aligned}
$$

\section{Interval analysis of free vibration}

Let $\mathbf{W}^{I}$ is an $n$-dimensional interval vector of uncertain material parameters or the load, if uncertainty of the density of the plate is not considered, namely, the matrix $\mathbf{M}$ is independent of $\mathbf{W}^{I}$, and Eq. (7) can be rewritten as follows:

$$
\left[-\mathbf{A}\left(W^{I}\right)+\frac{\eta}{2}\left(\mathbf{R}\left(W^{I}\right)+\mathbf{R}^{T}\left(W^{I}\right)+\frac{\eta}{2} \mathbf{G}\left(W^{I}\right)\right)-\omega^{2} \mathbf{M}\right] \mathbf{U}\left(W^{I}\right)=0 .
$$

Interval variable $\mathbf{W}^{I}$ can also be expressed as:

$$
\mathbf{W}^{I}=\left\{\mathbf{W} \mid \mathbf{W}=\mathbf{W}^{c}+[-1,1] \mathbf{W}^{r}, \mathbf{W} \in R^{n}\right\},
$$

where $\mathbf{W}^{c}$ is the interval midpoint value, $\mathbf{W}^{r}$ the interval radius:

$$
\mathbf{W}^{c}=\frac{\mathbf{W}^{U}+\mathbf{W}^{L}}{2},
$$


$\mathbf{W}^{r}=\frac{\mathbf{W}^{U}-\mathbf{W}^{L}}{2}$

where $\mathbf{W}^{L}$ denotes the lower bound, and $\mathbf{W}^{U}$ the upper bound.

The uncertainty level of the interval variable is defined as:

$W^{\text {level }}=\frac{W^{r}}{W^{c}} \times 100 \%$

The modal vector $U^{I}$ can be expanded into the first-order Taylor series:

$\mathbf{U}^{I}=U\left(\mathbf{W}^{I}\right)=\mathbf{U}\left(\mathbf{W}^{c}\right)+\sum_{j=1}^{n} \frac{\partial \mathbf{U}\left(\mathbf{W}^{c}\right)}{\partial W_{j}}\left(W_{j}^{I}-W_{j}^{c}\right)$.

The midpoint and interval radius of $\mathbf{U}^{I}$ can be obtained from Eq. (14):

$$
\begin{aligned}
& \mathbf{U}^{c}=\mathbf{U}\left(W_{j}^{c}\right), \\
& \mathbf{U}^{r}=\sum_{j=1}^{n}\left|\frac{\partial \mathbf{U}\left(\mathbf{W}^{c}\right)}{\partial W_{j}}\right| W_{j}^{r},
\end{aligned}
$$

where:

$$
\begin{aligned}
& \mathbf{U}^{c}=\frac{\mathbf{U}^{L}+\mathbf{U}^{U}}{2}, \\
& \mathbf{U}^{r}=\frac{\mathbf{U}^{L}-\mathbf{U}^{U}}{2},
\end{aligned}
$$

where $\mathbf{U}^{L}$ denotes the lower bound, and $\mathbf{U}^{U}$ the upper bound.

Vibration natural frequencies of the plate can also be expanded into the first-order Taylor series:

$\omega_{n i}^{I}=\omega_{n i}\left(\mathbf{W}^{c}\right)+\sum_{j=1}^{n} \frac{\partial \omega_{n i}\left(\mathbf{W}^{c}\right)}{\partial W_{j}}\left(W_{j}^{I}-W_{j}^{c}\right)$.

The midpoint and interval radius of $\omega_{n i}^{I}$ can be obtained from Eq. (19):

$\omega_{n i}^{c}=\omega_{n i}\left(\mathbf{W}^{c}\right)$,

$\omega_{n i}^{r}=\sum_{j=1}^{n}\left|\frac{\partial \omega_{n i}\left(\mathbf{W}^{c}\right)}{\partial W_{j}}\right| W_{j}^{r}$

In order to obtain $\partial \omega^{c} / \partial W_{j}$, Partial derivative both sides of Eq. (9) with respect to $W_{j}$ at the midpoint $\mathbf{W}^{c}$, we have:

$$
\begin{gathered}
{\left[-\frac{\partial \mathbf{A}\left(W^{c}\right)}{\partial W_{j}}+\frac{\eta}{2}\left(\frac{\partial \mathbf{R}\left(W^{c}\right)}{\partial W_{j}}+\frac{\partial \mathbf{R}^{T}\left(W^{c}\right)}{\partial W_{j}}+\frac{\eta}{2} \frac{\partial \mathbf{G}\left(W^{c}\right)}{\partial W_{j}}\right)-2 \omega^{c} \frac{\partial \omega^{c}}{\partial W_{j}} \mathbf{M}\right] \mathbf{U}\left(W^{c}\right)} \\
+\left[-\mathbf{A}\left(W^{c}\right)+\frac{\eta}{2}\left(\mathbf{R}\left(W^{c}\right)+\mathbf{R}^{T}\left(W^{c}\right)+\frac{\eta}{2} \mathbf{G}\left(W^{c}\right)\right)-\omega^{c 2} \mathbf{M}\right] \frac{\partial \mathbf{U}\left(W^{c}\right)}{\partial W_{j}}=0 .
\end{gathered}
$$


The two sides of Eq. (22) multiplied by $\mathbf{U}^{T}\left(W^{c}\right)$ has:

$$
\begin{aligned}
& \mathbf{U}^{T}\left(W^{c}\right)\left[-\frac{\partial \mathbf{A}\left(W^{c}\right)}{\partial W_{j}}+\frac{\eta}{2}\left(\frac{\partial \mathbf{R}\left(W^{c}\right)}{\partial W_{j}}+\frac{\partial \mathbf{R}^{T}\left(W^{c}\right)}{\partial W_{j}}+\frac{\eta}{2} \frac{\partial \mathbf{G}\left(W^{c}\right)}{\partial W_{j}}\right)-2 \omega^{c} \frac{\partial \omega^{c}}{\partial W_{j}} \mathbf{M}\right] \mathbf{U}\left(W^{c}\right) \\
& +\mathbf{U}^{T}\left(W^{c}\right)\left[-\mathbf{A}\left(W^{c}\right)+\frac{\eta}{2}\left(\mathbf{R}\left(W^{c}\right)+\mathbf{R}^{T}\left(W^{c}\right)+\frac{\eta}{2} \mathbf{G}\left(W^{c}\right)\right)-\omega^{c} \mathbf{M}\right] \frac{\partial \mathbf{U}\left(W^{c}\right)}{\partial W_{j}}=0 .
\end{aligned}
$$

From Eq. (9), we have:

$$
\left[-\mathbf{A}\left(W^{c}\right)+\frac{\eta}{2}\left(\mathbf{R}\left(W^{c}\right)+\mathbf{R}^{T}\left(W^{c}\right)+\frac{\eta}{2} \mathbf{G}\left(W^{c}\right)\right)-\omega^{c^{2}} \mathbf{M}\right] \mathbf{U}\left(W^{c}\right)=0 .
$$

The transpose of Eq. (24) yields:

$$
\begin{aligned}
& \mathbf{U}^{T}\left(W^{c}\right)\left[-\mathbf{A}\left(W^{c}\right)+\frac{\eta}{2}\left(\mathbf{R}\left(W^{c}\right)+\mathbf{R}^{T}\left(W^{c}\right)+\frac{\eta}{2} \mathbf{G}\left(W^{c}\right)\right)-\omega^{c^{2}} \mathbf{M}\right]^{T} \\
& \quad=\mathbf{U}^{T}\left(W^{c}\right)\left[-\mathbf{A}\left(W^{c}\right)+\frac{\eta}{2}\left(\mathbf{R}\left(W^{c}\right)+\mathbf{R}^{T}\left(W^{c}\right)+\frac{\eta}{2} \mathbf{G}\left(W^{c}\right)\right)-\omega^{c^{2}} \mathbf{M}\right]=0_{1 \times 5}
\end{aligned}
$$

Substitution of Eq. (25) into Eq. (23), yields the following equation:

$$
\begin{aligned}
& \mathbf{U}^{T}\left(W^{c}\right)\left[-\frac{\partial \mathbf{A}\left(W^{c}\right)}{\partial W_{j}}+\frac{\eta}{2}\left(\frac{\partial\left(\mathbf{R}\left(W^{c}\right)+\mathbf{R}^{T}\left(W^{c}\right)\right)}{\partial W_{j}}+\frac{\eta}{2} \frac{\partial \mathbf{G}\left(W^{c}\right)}{\partial W_{j}}\right)-2 \omega^{c} \frac{\partial \omega^{c}}{\partial W_{j}} \mathbf{M}\right] \mathbf{U}\left(W^{c}\right) \\
& \quad=0 .
\end{aligned}
$$

$\partial \omega^{c} / \partial W_{j}$ can be obtained from Eq. (26):

$\frac{\partial \omega^{c}}{\partial W_{j}}=\frac{\mathbf{U}^{T}\left(W^{c}\right)\left[-\frac{\partial \mathbf{A}\left(W^{c}\right)}{\partial W_{j}}+\frac{\eta}{2}\left(\frac{\partial \mathbf{R}\left(W^{c}\right)}{\partial W_{j}}+\frac{\partial \mathbf{R}^{T}\left(W^{c}\right)}{\partial W_{j}}+\frac{\eta}{2} \frac{\partial \mathbf{G}\left(W^{c}\right)}{\partial W_{j}}\right)\right] \mathbf{U}\left(W^{c}\right)}{2 \omega^{c} \mathbf{U}^{T}\left(W^{c}\right) \mathbf{M U}\left(W^{c}\right)}$

$\partial \mathbf{U}\left(W^{c}\right) / \partial W_{j}$ can be obtained by substitution of Eq. (27) into Eq. (22) and then solution of Eq. (23).

\section{Numerical example and discussion}

For all the subsequent numerical examples, the interval midpoint values of the uncertain material parameters are given in Appendix A. The length of the plate is $a=0.8 \mathrm{~m}$, the width $b=0.5 \mathrm{~m}$, and the thickness $h=0.05 \mathrm{~m}$. The exponential factor $\eta=-10,-5,0.5,10$.

In the paper, the following dimensionless parameters are employed:

$$
\begin{aligned}
& \bar{x}=\frac{x}{h}, \quad \bar{y}=\frac{y}{h}, \quad \bar{z}=\frac{z}{h}, \quad \bar{u}_{1}=\frac{C_{1212} u_{1}}{f_{0} h}, \quad \bar{u}_{2}=\frac{C_{1212} u_{2}}{f_{0} h}, \quad \bar{u}_{3}=\frac{C_{1212} u_{3}}{f_{0} h}, \\
& \bar{\varphi}=\frac{C_{1212} g_{s}}{f_{0} h e_{s}} \varphi, \quad \bar{\psi}=\frac{C_{1212} \mu_{s}}{f_{0} h q_{s}} \psi, \quad \bar{\omega}=\frac{\omega}{\sqrt{\frac{h C_{1212}}{\rho_{0}}}}
\end{aligned}
$$

where $g_{s}=10^{-10} \mathrm{As} / \mathrm{Vm}, e_{s}=\mathrm{C} / \mathrm{m}^{2}, \mu_{s}=10^{-6} \mathrm{Vs} / \mathrm{Am}, e_{s}=\mathrm{Vs} / \mathrm{m}^{2}, C_{1212}=14.4 \mathrm{GPa}$, $\rho_{0}=7454 \mathrm{Kg} / \mathrm{m}^{3}, f_{0}=1 \mathrm{~Pa}, h=0.05 \mathrm{~m}$.

Case I. The elastic parameters are considered as uncertain, the uncertainty level $\pm 1 \%$ off from 
the midpoints of the elastic parameters is investigated. The numerical results shows that the midpoint value and uncertainty level of the natural frequencies is symmetric about $\eta=0$ for $\eta=-10,-5,0.5,10$.

Therefore, Uncertainty level of the natural frequencies $(m=1, n=1)$ is only shown in Table 1 for $\eta=0.5,10$.

Table 1. Uncertainty level of the dimensionless natural frequencies $(m=1, n=1)$

\begin{tabular}{|c|c|c|}
\hline$\eta=0$ midpoint $(\mathrm{Hz})($ level $)$ & $\eta=5$ midpoint $(\mathrm{Hz})($ level $)$ & $\eta=10$ midpoint $(\mathrm{Hz})($ level $)$ \\
\hline $7.910(0.50 \%)$ & $9.083(1.19 \%)$ & $10.147(3.41 \%)$ \\
\hline $8.805(0.08 \%)$ & $9.489(0.73 \%)$ & $12.752(1.57 \%)$ \\
\hline $16.770(0.07 \%)$ & $17.073(0.33 \%)$ & $18.072(1.31 \%)$ \\
\hline
\end{tabular}

It can be seen from Table 1 that no matter how much the exponential factor is, uncertainty level of the dimensionless natural frequencies will decrease with increase of the dimensionless natural frequencies. For the same order dimensionless natural frequency, uncertainty level of the dimensionless natural frequencies will increase with the exponential factor.

Case II. The piezomagnetic coefficients are considered as uncertain, the uncertainty level $\pm 1 \%$ off from the midpoints of the piezomagnetic coefficients is investigated. The numerical results show that the midpoint value and uncertainty level of the dimensionless natural frequencies is symmetric about $\eta=0$ for $\eta=-10,-5,0.5,10$. Therefore, Uncertainty level of the dimensionless natural frequencies $(m=1, n=1)$ is only shown in Table 2 for $\eta=0.5,10$.

Table 2. Uncertainty level of the dimensionless natural frequencies $(m=1, n=1)$

\begin{tabular}{|c|c|c|}
\hline$\eta=0$ midpoint $(\mathrm{Hz})($ level $)$ & $\eta=5$ midpoint $(\mathrm{Hz})($ level $)$ & $\eta=10$ midpoint $(\mathrm{Hz})($ level $)$ \\
\hline $7.910\left(1.32 \times 10^{-7} \%\right)$ & $9.083\left(1.90 \times 10^{-7} \%\right)$ & $10.147\left(8.89 \times 10^{-8} \%\right)$ \\
\hline $8.805(0.00 \%)$ & $9.489\left(4.63 \times 10^{-7} \%\right)$ & $12.752\left(1.20 \times 10^{-6} \%\right)$ \\
\hline $16.770(0.00 \%)$ & $17.073\left(2.89 \times 10^{-8} \%\right)$ & $18.072\left(8.13 \times 10^{-8} \%\right)$ \\
\hline
\end{tabular}

It can be seen from Table 2 that no matter how much the exponential factor is, uncertainty level of the dimensionless natural frequencies is so small that it can be ignored. This can be explained that the piezomagnetic coefficients does not cause a change in the mass of the plate, and it has only a very small effect on the stiffness of the plate. Therefore, the dimensionless natural frequencies of the plate mainly determined by elastic parameters and mass of the plate.

Case III. The piezoelectric coefficients are considered as uncertain, the uncertainty level $\pm 1 \%$ off from the midpoints of the piezoelectric coefficients is investigated. The numerical results show that the midpoint value and uncertainty level of the dimensionless natural frequencies is symmetric about $\eta=0$ for $\eta=-10,-5,0.5,10$. Therefore, Uncertainty level of the dimensionless natural frequencies $(m=1, n=1)$ is only shown in Table 3 for $\eta=0,5,10$.

Table 3. Uncertainty level of the dimensionless natural frequencies $(m=1, n=1)$

\begin{tabular}{|c|c|c|}
\hline$\eta=0$ midpoint $(\mathrm{Hz})($ level $)$ & $\eta=5$ midpoint $(\mathrm{Hz})($ level $)$ & $\eta=10$ midpoint $(\mathrm{Hz})($ level $)$ \\
\hline $7.910\left(1.77 \times 10^{-9} \%\right)$ & $9.083\left(2.54 \times 10^{-9} \%\right)$ & $10.147\left(1.1 .9 \times 10^{-9} \%\right)$ \\
\hline $8.805(0.00 \%)$ & $9.489\left(6.18 \times 10^{-9} \%\right)$ & $12.752\left(1.60 \times 10^{-8} \%\right)$ \\
\hline $16.770(0.00 \%)$ & $17.073\left(3.86 \times 10^{-10} \%\right)$ & $18.072\left(1.09 \times 10^{-9} \%\right)$ \\
\hline
\end{tabular}

It can be seen from Table 3 that no matter how much the exponential factor is, uncertainty level of the dimensionless natural frequencies is so small that it can be ignored. There is the same explanation as the piezomagnetic coefficients.

It can also be seen from comparison between table 2 and table 3 that for the same uncertainty level off from the midpoints, the piezomagnetic coefficients can cause much larger uncertainty level of the dimensionless natural frequencies than the piezoelectric coefficients. This is the cause that the piezomagnetic has a greater influence on the stiffness of the plate than the piezoelectric coefficient. 


\section{Conclusions}

The elastic, electric and magnetic parameters are regarded as interval variables. Interval analysis of electric-magneto-elastic functionally graded plate are carried out by introducing the interval algorithm into the dynamic equations.

The numerical result shows:

1) The elastic parameters are considered as uncertain, no matter how much the exponential factor is, uncertainty level of the dimensionless natural frequencies will decrease with increase of the dimensionless natural frequencies. For the same order, dimensionless natural frequency, uncertainty level of the dimensionless natural frequencies will increase with increase of the exponential factor.

2) The piezomagnetic coefficients are considered as uncertain, no matter how much the exponential factor is, uncertainty level of the dimensionless natural frequencies is so small that it can be ignored. This can be explained that the piezomagnetic coefficients does not cause a change in the mass of the plate, and it has only a very small effect on the stiffness of the plate.

3) The piezoelectric coefficients are considered as uncertain, no matter how much the exponential factor is, uncertainty level of the dimensionless natural frequencies is so small that it can be ignored.

\section{Acknowledgement}

The work is supported by Natural Science Foundation of China under the Grant Number 11372109 and 11302159.

\section{References}

[1] Akbarzadeh A. H., Chen Z. T. Magnetoelectroelastic behavior of rotating cylinders resting on an elastic foundation under hygrothermal loading. Smart Material Structures, Vol. 20, Issue 6, 2011, p. $1645-1648$.

[2] Bishay P. L., Sladek J., Sladek V., Atluri S. N. Analysis of functionally graded magnetoelectro-elastic composites using hybrid/mixed finite elements and node-wise material properties. Computers, Materials and Continua, Vol. 29, Issue 3, 2012, p. 213-262.

[3] Saadatfar M., Aghaie-Khafri M. Hygrotherm-magneto-electro-elastic analysis of a functionally graded magneto-electro-elastic hollow sphere resting on an elastic foundation. Smart Material Structures, Vol. 23, Issue 3, 2014, p. 35004-35016.

[4] Li Yansong, Zhang Jingjun Free vibration analysis of magneto-electro-elastic plate resting on a Pasternak foundation. Smart Material Structures, Vol. 23, Issue 2, 2014, p. 204-214.

[5] Razavi Soheil, Shooshtari Alireza Free vibration analysis of a magneto-electro-elastic doubly-curved shell resting on a Pasternak-type elastic foundation. Smart Material Structures, Vol. 23, Issue 10, 2014, p. $64-75$.

[6] Shooshtari A., Razavi S. Linear and nonlinear free vibration of a multilayered magneto-electro-elastic doubly-curved shell on elastic foundation. Composites Part B Engineering, Vol. 78, 2015, p. 95-108.

[7] Niraula O. P., Wang B. L. A magneto-electro-elastic material with a penny-shaped crack subjected to temperature loading. Acta Mechanica, Vol. 187, Issue 1, 2006, p. 151-168.

[8] Chen Xiaohong Energy release rate and path-independent integral in dynamic fracture of magneto-electro-thermo-elastic solids. International Journal of Solids and Structures, Vol. 46, Issue 13, 2009, p. 2706-2711.

[9] Feng W. J., Pan E., Xu Wang Dynamic fracture analysis of a penny-shaped crack in a magnetoelectroelastic layer. International Journal of Solids and Structures, Vol. 44, Issue 24, 2004, p. 7955-7974.

[10] Chen Hui, Yu Wenbin A multiphysics model for magneto-electro-elastic laminates. European Journal of Mechanics A/Solids, Vol. 47, 2014, p. 23-44.

[11] Han X., Jiang C., Gong S., Huang Y. H. Transient waves in composite-laminated plates with uncertain load and material property. International Journal for Numerical Methods in Engineering, Vol. 75, 2008, p. 253-274. 
[12] Li Fangyi, Luo Zhen, Sun Guangyong Reliability-based multi objective design optimization under interval uncertainty. CMES, Vol. 74, Issue 1, 2011, p. 39-64.

[13] Jiang C., Han X., Liu G. R., Liu G. P. A nonlinear interval number programming method for uncertain optimization problems. European Journal of Operational Research, Vol. 188, 2008, p. 1-13.

[14] Kang Zhan, Luo Yangjun, Li Alex On non-probabilistic reliability-based design optimization of structures with uncertain-but-bounded parameters. Structural Safety, Vol. 33, 2011, p. 196-205.

[15] Wu J., Zhao Y., Chen S. An improved interval analysis method for uncertain structures. Structural Engineering and Mechanics, Vol. 20, Issue 6, 2005, p. 713-726.

\section{Appendix}

The midpoint values of magnetic, electric and material parameters are given by:

$$
\begin{aligned}
& \mathbf{C}^{0}=\left[\begin{array}{cccccc}
79.7 & 35.8 & 35.8 & 0 & 0 & 0 \\
35.8 & 79.7 & 35.8 & 0 & 0 & 0 \\
35.8 & 35.8 & 66.8 & 0 & 0 & 0 \\
0 & 0 & 0 & 17.2 & 0 & 0 \\
0 & 0 & 0 & 0 & 14.4 & 0 \\
0 & 0 & 0 & 0 & 0 & 14.4
\end{array}\right] \mathrm{GPa} \text {, } \\
& \boldsymbol{\mu}^{0}=\left[\begin{array}{ccc}
5.4 & 0 & 0 \\
0 & 5.4 & 0 \\
0 & 0 & 5.4
\end{array}\right] \times 10^{-6} V S / A M \text {, } \\
& \mathbf{g}^{0}=\left[\begin{array}{ccc}
3.8 & 0 & 0 \\
0 & 3.8 & 0 \\
0 & 0 & 3.8
\end{array}\right] \times 10^{-10} A s / V m \text {, } \\
& e^{0}=\left[\begin{array}{cccccc}
0 & 0 & 0 & 0 & 10.5 & 0 \\
0 & 0 & 0 & 10.5 & 0 & 0 \\
-5.9 & -5.9 & 15.2 & 0 & 0 & 0
\end{array}\right] c / m^{2}, \\
& q^{0}=\left[\begin{array}{cccccc}
-5 & -5.9 & 15.2 & 0 & 0 & 0 \\
0 & 0 & 0 & 0 & 108.3 & 0 \\
-60.9 & -60.9 & 156.8 & 0 & 0 & 0
\end{array}\right] \mathrm{Vs} / \mathrm{m}^{2} \text {, } \\
& \rho^{0}=\frac{7454 \mathrm{~kg}}{m^{3}}, \quad \alpha^{0}=\left[\begin{array}{ccc}
3.0 & 0 & 0 \\
0 & 3.0 & 0 \\
0 & 0 & 3.0
\end{array}\right] \times 10^{-12} \mathrm{Ns} / \mathrm{Vm} \text {. }
\end{aligned}
$$

